\title{
Evaluating and assessing the prevalence of bedside ultrasound in emergency departments in China
}

\author{
Di Shi ${ }^{1}$, Joseph H. Walline ${ }^{2}$, Xuezhong Yu ${ }^{1}$, Jun $\mathrm{Xu}^{1}$, Priscilla P. Song ${ }^{3}$, Huadong Zhu ${ }^{1}$ \\ ${ }^{1}$ Department of Emergency Medicine, Peking Union Medical College Hospital, Beijing 100730, China; ${ }^{2}$ Department of Surgery, Division of \\ Emergency Medicine, Saint Louis University Hospital, Saint Louis, MO, USA; ${ }^{3}$ Department of Anthropology, Washington University in St. Louis, \\ Saint Louis, MO, USA \\ Contributions: (I) Conception and design: All authors; (II) Administrative support: All authors; (III) Provision of study materials or patients: All \\ authors; (IV) Collection and assembly of data: D Shi, J Xu; (V) Data analysis and interpretation: D Shi, J Xu; (VI) Manuscript writing: All authors; (VII) \\ Final approval of manuscript: All authors. \\ Correspondence to: Jun Xu, MD. Department of Emergency Medicine, Peking Union Medical College Hospital, No. 1, Shuai Fu Yuan, Dong Cheng \\ district, Beijing 100730, China. Email: xujunfree@126.com.
}

Background: To survey the prevalence of bedside ultrasound assessment in emergency departments (EDs) in China.

Methods: We designed an online survey for emergency physicians based in the China. The questionnaire included sixteen items querying common ED bedside ultrasound practices. Respondents were recruited via weblinks sent through social media and a popular Chinese emergency medicine website. Survey data was collected from April through June, 2016.

Results: Four hundred and twenty-eight physicians responded to this survey; more than $80 \%$ of respondents reported working clinically in the ED. Ninety-eight percent of respondents agreed on the clinical importance and value of bedside ultrasound. However, less than half of participants' EDs had ultrasound devices, and less than half of the respondents said they knew how to perform bedside ultrasound. Less than $20 \%$ of respondents reported having had formal training in bedside ultrasound.

Conclusions: There is a strong interest in bedside ultrasound in Chinese EDs. Emergency physicians participating in this study considered bedside ultrasound a necessary skill, but, because there is a lack of training, most emergency physicians reported they did not know how to perform bedside ultrasonography. There is likely an acute desire and need for bedside ultrasound training for Chinese emergency physicians.

Keywords: Emergency medicine; bedside ultrasound; prevalence

Submitted Dec 18, 2017. Accepted for publication Apr 07, 2018.

doi: $10.21037 /$ jtd.2018.04.88

View this article at: http://dx.doi.org/10.21037/jtd.2018.04.88

\section{Introduction}

The practice of bedside ultrasound was pioneered in intensive care units by cardiologists for critical patients in the 1980s (1). In the past three decades, the bedside ultrasound exam has become a key assessment performed not only by intensivist physicians but also by emergency physicians due to its non-invasive and portable nature along with the benefits of avoiding ionizing radiation and ultrasound's relative low cost became a rapid assessment of patients for diagnosis and treatment (2). In the United States, bedside ultrasonography was identified as one of the essential skills for emergency physicians in 2011 (3) and its integration into the core curriculum of all emergency medicine residency training programs has been recommended by the American College of Emergency Physicians (ACEP) since 2008 (4).

In China, bedside ultrasound has been used since 2006 in emergency departments (ED) for the assessment of abdomen and guidance for vascular procedures (5). With 
the continuing development of EDs in China, increasing numbers of departments have their own devices, and more and more emergency physicians have begun to realize the usefulness of bedside ultrasound for rapidly assessing ED patients. A bedside ultrasound training course hosted by Peking Union Medical College Hospital's Emergency Medicine Department in 2015 attracted over hundreds of participants from over 12 provinces, suggesting anecdotally the intense interest of emergency physicians around China in learning about bedside ultrasound.

Ten years after its first use, how widespread is ED bedside ultrasound use in China? To what degree is bedside ultrasound felt to be a necessary and important skill for the Chinese emergency physician? How confident are Chinese emergency physicians in their ultrasound training? We sought to look not only at Beijing, but also at China as a whole. We organized an online questionnaire to develop a comprehensive understanding of the state of bedside ultrasound practice more broadly in China.

\section{Methods}

\section{Overview}

We conducted a cross-sectional study by enrolling emergency physicians from EDs throughout mainland China between April 1 and June 30, 2016. The Institutional Review Board of Peking Union Medical College Hospital (No. S-K453) approved this study.

\section{Survey content and administration}

We designed an online survey using the REDCap system to query how ultrasound assessment was practiced in the EDs in which participants worked (6). Questions were formulated based on clinical experience, review of the existing literature, and discussions with emergency clinicians. To ensure the validity, three senior emergency physicians with both extensive ultrasound experiences and prior training in survey design reviewed each question for relevance and clarity. Questions were pilot-tested among clinicians based in a major tertiary care hospital in Beijing and subsequently honed. The questionnaire was posted online at "ER-Vision", which is the official website of the emergency medicine division of the Chinese Medical Doctor Association. Participants filled-in the electronic questionnaire anonymously. Questionnaires consisted of sixteen items consisting of single-choice, multiple-choice, or branching questions as well as open-ended questions asking about basic demographic data, participants' background in using bedside ultrasound, their previous ultrasound training experiences, areas of ultrasound utilized in their own clinical practice and their suggestions for future bedside ultrasound training programs.

Demographic information collected included which types of hospitals they worked in, and the number of years they have been in practice. Respondents were not offered any incentives to participate in the survey.

\section{Statistical analysis}

A dedicated statistician worked with our research group throughout the survey design and analysis stages of this project. The survey data were subsequently analyzed by descriptive statistics. In order to examine the association between participant's response and physician seniority and hospital characteristics, the comparisons were made by fisher's exact test with statistical significance level set at $\mathrm{P}<0.05$.

\section{Results}

This survey had 428 respondents. They came from 30 (out of 34) province-level administrative divisions regions of mainland China, with 109 (25.5\%) from Beijing. One hundred and thirty-seven (32\%) were working in tertiary hospitals of prefecture-level cities (e.g., Beijing, Shanghai, Chongqing, and Tianjin), 216 (50.5\%) were from provincial tertiary hospitals. $344(80.4 \%)$ of participants were emergency physicians. The remaining 84 (19.6\%) were from emergency ICU or internal medicine departments who were working in ED. Participants included both senior and junior physicians: department chairs, vice-chairs, senior and junior attending physicians, as well as resident physicians.

Two hundred and thirty-five (54.9\%) participants did not have a bedside ultrasound device in their ED with two did not answer to the question. Among 191 (44.6\%) participants whose department had their own ultrasound device(s), the availabilities of ultrasound devices seemed to be variable between institutions. One hundred and twentyfour $(64.9 \%$ of 191) participants indicated their department owned only one ultrasound device, and 34 (17.9\% of 191) participants had access to more than three devices in their ED. Most of the devices were purchased new with only $15.7 \%$ of such ultrasound devices were retired from 
Table 1 Assessments and protocols used by physicians with bedside ultrasound for patient's care

\begin{tabular}{|c|c|c|c|}
\hline Assessment/protocol & Counts & $\begin{array}{l}\text { Percentage (among entire } \\
\text { cohort, total } \mathrm{N}=428 \text { ) }\end{array}$ & $\begin{array}{l}\text { Percentage (among only physicians currently practicing } \\
\text { bedside ultrasound, total } \mathrm{N}=186 \text { ) }\end{array}$ \\
\hline IV access & 163 & 38.3 & 87.6 \\
\hline Echocardiography & 125 & 29.3 & 67.2 \\
\hline Shock & 94 & 22.1 & 50.5 \\
\hline Vascular & 80 & 18.8 & 43.0 \\
\hline Short of breath & 82 & 19.2 & 44.1 \\
\hline Lumbar puncture & 11 & 2.6 & 5.9 \\
\hline $\begin{array}{l}\text { Transesophageal- } \\
\text { echocardiography }\end{array}$ & 5 & 1.2 & 2.7 \\
\hline
\end{tabular}

radiology departments. 136 (71.2\% of 191) participants had portable bedside ultrasound. 105 (55\% of 191) participants' department own devices with three probes and only 27 (14.1\% of 191) had more than three probes.

Almost all participants 421 (98.4\%) agreed that bedside ultrasound was necessary and valuable for emergency medicine patient diagnosis, management and reassessment. in addition, there were only $19.9 \%$ who considered that ultrasound could be replaced by another skill for assessing ED patients. However, of all the participants in the survey only $43.5 \%$ admitted to performing bedside ultrasound evaluations themselves. The most frequently performed bedside ultrasound protocols and assessments are ultrasound guided central-line placement, paracentesis or thoracentesis, followed by echocardiography, lung and FAST assessments. Lumbar puncture and transesophagealechocardiography were least performed procedures. More details are enumerated in Table 1 .

Of 186 practitioners who admitted to performing ultrasound assessments on patients, most performed procedures are the "FAST" exams (139 participants, 74.7\%) (7), the "BLUE" protocol $(70,37.6 \%)(8)$ and the "RUSH" protocol $(37,19.9 \%)$ for hemodynamic assessments. while $29(15.6 \%)$ did the "FALLS" protocol for undifferentiating shock patient diagnosis and management $(9,10)$.
The participants perceived the biggest challenge in ultrasound clinical practice was how to get images with better quality. Other problems included: patient positioning, adjustment of probe frequency, lack of familiarity with the advanced functions for ultrasound evaluation (e.g., calculating tools).

Of all the 186 physicians who have practiced bedside ultrasound in the emergency room, only 36 (19.4\%) participants had received formal systematic ultrasound training. One hundred and two $(54.8 \%)$ physicians practiced after self-learning and 45 (24.2\%) had continuous education in their institution's radiology department. Participants reported more than 20 kinds of ultrasound training courses that they took part in. Government and hospitals organized the most training courses, and most of the courses were workshop-style and a minority were lecture-based. Sixty $(32.3 \%)$ participants stated that they only received training less than 10 hours before working on their own patients while only $34(18.3 \%)$ had more than 60 hours of training. Seventy-seven $(41.4 \%)$ of practitioners evaluated images themselves without additional tools, $42(22.6 \%)$ worked with calculating tools and $67(36 \%)$ used comparisons to additional formal studies.

To understand how well ED-performed ultrasound assessment was viewed in their institution, we designed a 
question that asked if a surgeon at their institution would use the ED-performed FAST outcome in making an operative decision, to which $23.1 \%$ of participants choose 'yes'.

To examine how physicians' seniority and type of institutions could have impacted participants' experiences with ER ultrasound, stratified analysis compared the attending, fellow and residents physicians, and participants from providential tertiary, local tertiary and community hospitals. Overall, senior physicians are more likely to have past experiences of various ultrasound facilitated procedures as compared with fellows. Community hospital physicians are less likely to have experiences with or be familiar with ultrasound facilitated procedures, and more likely to have difficulties with quality of the image and rely most on eyeball measurement rather than instruments (Table 2).

\section{Discussion}

This study is the first, largest and most comprehensive study to date looking at the prevalence of bedside ultrasound practice in Chinese mainland EDs. We recruited emergency physicians of different seniorities (residents, fellows, and attending physicians) and from different institutions (provincial tertiary, local tertiary and community hospitals). The staffing mode in Chinese ED is consisting of rotating specialist from internal medicine and surgery, as well as emergency physicians. The rotating specialist covered part of urgent patient care. For better solving crowed issue and severe patient care in ED, some Chinese ED established emergency intensive care unit in the past ten years. As many Chinese ED also have their own 'emergency' intensive care unit, our study also recruited intensivists who work in the emergency intensive care unit.

In the past decade, bedside ultrasound has become one of the most valuable skills for emergency physicians. Almost all of the study participants agreed on the necessity of ultrasound in clinical practice. However, the availabilities of ultrasound devices and ultrasound utilization were still low. Less than $50 \%$ of participant's department had a bedside ultrasound device. Despite the fact that over half of participants were attending and associate professors who were the most active in clinical procedures, less than $50 \%$ of respondents had bedside ultrasound experience.

Among the participants who had performed bedside ultrasound procedures, bedside ultrasound was most likely to be used for paracentesis, shock assessments and the FAST exam. Participants rarely used ultrasound to evaluate patients for abdominal, lung or cardiac abnormalities. Some of the practitioners did perform FAST or eFAST protocols but less than a quarter of the relevant surgeons would have taken the outcome into consideration in making an operative decision.

Senior physicians and residents (as most of them were from large tertiary hospitals) were more likely to prior experiences with bedside ultrasound procedures and use instrument measurement rather than eyeball or hand measurement. On the other hand, fellows and participants from community hospitals were the least likely to have ultrasound experiences and relied more on eyeball in measurement. It is possible that the residents from tertiary hospitals were more likely to receive bedside ultrasound training or accumulate more experiences due to more convenient access to up-to-date ultrasound techniques, despite the fact they are more junior when compared with fellows from other lower tier hospitals.

It was also evident that training of bedside ultrasound was inadequate. More than half of participants didn't have any training courses or even self-study opportunities through affiliated radiology departments. Few respondents had enrolled in ultrasound training courses but none of training courses were designed for emergency physicians. Most practitioners had very little training before assessing their own patients and they were quite concerned about having high quality images or had difficulties in measurement tools.

This study achieved its goals of surveying a large crosssection of mainland Chinese emergency physicians. However, there were still some limitations. As an online survey, it is difficult to be certain the possible total number of physicians offered the survey (i.e., to calculate a response rate). The convenient sampling of the participants could not be weighted to reflect the overall ED practitioners across the country. Differentiating emergency physician respondents from non-ED providers depended on selfreported answers to the survey, and were not independently verifiable. More advanced studies should be done in the future to systematically sample within different institution characteristics and properly weigh on subgroups to really represent the national statistics of all ED in mainland China. We expect there are larger gaps of ultrasound adoption and greater need for effective training programs in the more remote, community hospitals. Follow-up studies which quantitatively measure the performance of ED physicians are also needed to better ascertain the current bedside ultrasound practice in ED.

In conclusion, this study surveyed the current experiences of ED physicians toward bedside ultrasound 
Table 2 participants' ultrasound experiences by physician seniority and hospital characteristics

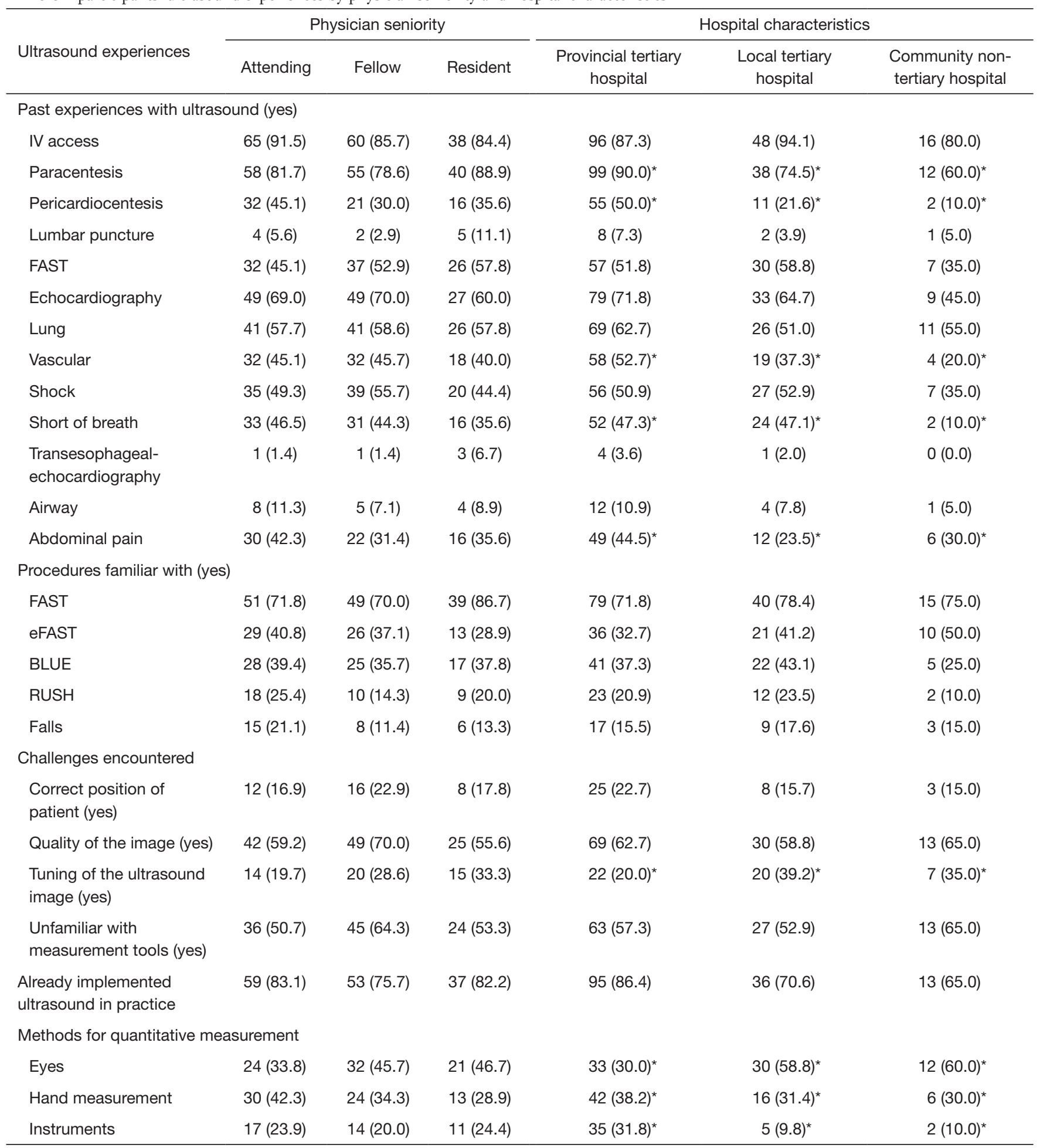

Data were shown as the numbers and percentage of participants within each column subgroup; the cell with * indicate there were statistical significant differences in the distribution between subgroups regarding that characteristics $(P<0.05)$. 
training in mainland Chinese EDs. There was a lacking of past experiences with bedside ultrasound and training among study sample. Participants recognized the importance to learn and practice bedside ultrasound skills in clinical practice, but there is no standard training program developed yet for emergency physicians. We recommend further standardization and broadening the availability of bedside ultrasound education in China in the future, to meet the needs of practicing emergency physicians.

\section{Acknowledgements}

None.

\section{Footnote}

Conflicts of Interest: The authors have no conflicts of interest to declare.

Ethical Statement: The Institutional Review Board of Peking Union Medical College Hospital (No. S-K453) approved this study.

\section{References}

1. Vieillard-Baron A, Slama M, Cholley B, et al.

Echocardiography in the intensive care unit: from evolution to revolution? Intensive Care Med 2008;34:243-9.

2. Jardin F, Valtier B, Beauchet A, et al. Invasive monitoring

Cite this article as: Shi D, Walline JH, Yu X, Xu J, Song PP, Zhu H. Evaluating and assessing the prevalence of bedside ultrasound in emergency departments in China. J Thorac Dis 2018;10(5):2685-2690. doi: 10.21037/jtd.2018.04.88 combined with two dimensional echocardiographic study in septic shock. Intensive Care Med 1994;20:550-4.

3. 2011 EM Model Review Task Force, Perina DG, Brunett CP, et al. The 2011 model of the clinical practice of emergency medicine. Acad Emerg Med 2012;19:e19-40.

4. American College of Emergency Physicians. Emergency ultrasound guidelines. Ann Emerg Med 2009;53:550-70.

5. Zhu H, Liu J, Liu Y, et al. Effect of bedside ultrasound performed by emergency physician on the trauma patients. Chin J Crit Care Med 2009;29:557-9.

6. Harris PA, Taylor R, Thielke R, et al. Research electronic data capture (REDCap)--a metadata-driven methodology and workflow process for providing translational research informatics support. J Biomed Inform 2009;42:377-81.

7. Shaukat NM, Copeli N, Desai P. The focused assessment with sonography for trauma (FAST) examination and pelvic trauma: indications and limitations. Emerg Med Pract 2016;18:1-20, 24; quiz 20-1.

8. Frankel HL, Kirkpatrick AW, Elbarbary M, et al. Guidelines for the appropriate use of bedside general and cardiac ultrasonography in the evaluation of critically Ill Patients-Part I: General Ultrasonography. Crit Care Med 2015;43:2479-502.

9. Perera P, Mailhot T, Riley D, et al. The RUSH exam: Rapid Ultrasound in SHock in the evaluation of the critically ill. Emerg Med Clin North Am 2010;28:29-56, vii.

10. Lichtenstein D. FALLS-protocol: lung ultrasound in hemodynamic assessment of shock. Heart Lung Vessel 2013;5:142-7. 